\title{
IMPLEMENTATION OF SIGMA METRICS FOR EVALUATION OF ANALYTICAL QUALITY IN CLINICAL BIOCHEMISTRY LABORATORY OF A TERTIARY CARE HOSPITAL
}

\author{
Jayati Roy Choudhury1, Sayari Banerjee², Indranil Chakraborty ${ }^{3}$
}

1 Demonstrator, Department of Biochemistry, College of Medicine and Sagore Dutta Hospital, Kolkata, West Bengal. 2Demonstrator, Department of Biochemistry, College of Medicine and Sagore Dutta Hospital, Kolkata, West Bengal. ${ }^{3}$ Professor \& HOD, Department of Biochemistry, College of Medicine and Sagore Dutta Hospital, Kolkata, West Bengal.

ABSTRACT
BACKGROUND
Amelioration of analytical quality in clinical laboratories is recognised as an emerging concern worldwide. Quality has been
described as conformance to the requirement of users and the satisfaction of their needs and expectations. Quality assurance is
need of the hour in all sectors including laboratory service. Six sigma provides a general methodology to describe performance on
sigma scale.
The present study was undertaken to evaluate the quality of the analytical performance of clinical biochemistry laboratory
with the help of sigma metrics.

\section{MATERIALS AND METHODS}

Internal quality control (QC) data for both levels of control, L1 (normal) and L2 (high) obtained from clinical biochemistry laboratory of College of Medicine and Sagore Dutta Hospital were analysed over a period of one year from July 2016 to July 2017. Laboratory mean, standard deviation(SD) and coefficient of variation (CV \%) were calculated for parameters like sugar, urea, creatinine, triglyceride, cholesterol, High Density Lipoprotein (HDL), Bilirubin, Aspartate Aminotransferase (AST), Alanine Aminotransferase (ALT), Alkaline Phosphatase (ALP), total protein, albumin and uric acid. Sigma factor was calculated using both levels of internal QC for the above-mentioned parameters.

\section{RESULTS}

Parameters such as ALP, Triglyceride (L2) and HDL with average sigma values ( $>6$ ) was considered as satisfactory. Parameters having average Sigma 3 - 6 relating to glucose, urea, creatinine (L2), ALT (L2), AST (L2), uric acid, Bilirubin total, Cholesterol (L1), Total protein and albumin were considered as acceptable, but needed close supervision.

We got sigma < 3 for parameters like ALT (L1), AST (L1), Creatinine (L1) and Cholesterol (L2) which were unacceptable.

\section{CONCLUSION}

Sigma metrics thus calculated can be used as a guide for planning quality control strategy. The findings of our study will emphasise the need for evaluation of ongoing quality assurance programme and adoption of corrective and preventive measure. Ideal analytical methodology, quality control material and quality control strategy should be introduced to achieve uniform six sigma standards across all laboratories.

\section{KEY WORDS}

Quality Control, Mean, Six Sigma, Coefficient of Variation, Tea.

HOW TO CITE THIS ARTICLE: Choudhury JR, Banerjee S, Chakraborty I. Implementation of sigma metrics for evaluation of analytical quality in clinical biochemistry laboratory of a tertiary care hospital. J. Evolution Med. Dent. Sci. 2018;7(21):2539-2542, DOI: $10.14260 /$ jemds/2018/571

\section{BACKGROUND}

Quality refers to satisfaction of the needs and expectations of the users or customers. Improvement in quality as well as reduction in cost in healthcare system is of utmost importance and also a basic need. Total Quality management in a clinical laboratory comprises of- 1) Quality Laboratory Process, 2) Quality Control, 3) Quality Assessment and 4) Quality Systems. Several new quality initiatives have been developed and implemented to ensure quality management, among these. Six Sigma is one of the most powerful tool to improve laboratory quality management. Six sigma metrics are being adopted as the universal measure of quality.

'Financial or Other Competing Interest': None.

Submission 11-04-2018, Peer Review 04-05-2018,

Acceptance 11-05-2018, Published 21-05-2018.

Corresponding Author:

Dr. Sayari Banerjee,

Department of Biochemistry,

College of Medicine and Sagore Dutta Hospital,

Kamarhati, Kolkata-700058, West Bengal.

E-mail: banerjee.sayari99@gmail.com

DOI: $10.14260 /$ jemds $/ 2018 / 571$

\section{(c) (i) $Ð$}

Six sigma provides a general methodology to describe performance on sigma scale.1,2 Any process in clinical biochemistry laboratory can be evaluated in terms of a sigma metric that describes how many Sigmas fit within the tolerance limits.

Two methods can be used to assess the process performance in terms of a sigma metric. One approach is to measure outcomes by inspection. The other approach is to measure variation and predict process performance. Measurement of outcome is done by calculating defects per million (DPM) and converting it into sigma metric. ${ }^{3}$

Quality in a laboratory is measured on the sigma scale with a criterion of $3 \sigma$ as the minimum allowable sigma for routine performance and a sigma of $6 \sigma$ was considered as world-class quality. ${ }^{2}$ The present day healthcare services are only functioning at 3 sigma and in some cases 4 sigma levels that translate roughly into 66,807 and 6,210 DPM opportunities respectively.4 Present study was conducted in Clinical Biochemistry Laboratory of College of Medicine and Sagore Dutta Hospital for one year. Aim of our study was to measure the sigma metrics of various parameters of our laboratory and to assess the error associated with it, so that 
we could implement proper quality control strategy to augment patient compliance as well as reduce cost.

We analysed sigma metrics of 13 parameters with autoanalyser EM 360. The study protocol was approved by Institutional Human Ethics Committee.

\section{MATERIALS AND METHODS}

This hospital-based cross-sectional study was performed in College of Medicine and Sagore Dutta Hospital after obtaining clearance by Institutional Ethics Committee. Study material used were internal quality control material, External quality control material, QC data from EM360 autoanalyser software. Various parameters that were scrutinised are sugar, urea creatinine, Triglyceride, cholesterol, HDL, Bilirubin, SGOT, SGPT, ALP, total protein, albumin and uric acid. Results of both levels of internal quality control (Normal L1 and High L2) were recorded.

Bias \% is calculated from External Quality Assurance Scheme (EQAS) with the formula: Bias\%= [(Our lab result Peer group mean) / (Peer group mean) $]^{*} 100$.

CV\% is calculated from Internal Quality Control (IQC) data with the formula $\mathrm{CV} \%=(\mathrm{SD} / \mathrm{Mean}) \mathrm{x} 100$. Total allowable errors will be followed as per Clinical Laboratory Improvement Amendments (CLIA) guidelines. ${ }^{5}$

Sigma metrics were calculated from $\mathrm{CV}$, percentage bias and total allowable error. The sigma metrics for the various analytes was calculated by the following equation. ${ }^{1}$

The Equation for Calculating Sigma is =

$\sum(\sigma)=\left(T E_{a}-\right.$ bias $) / C V$

[TEa - total allowable error, $\mathrm{CV}$ —coefficient of variation].

Statistical analysis was done using R software.

\section{RESULTS}

In our study we find that parameters such as ALP, Triglyceride (L2) and HDL, the sigma values were found to be more than 6. For parameters- glucose, creatinine, triglycerides (L1), urea, SGPT (L2), SGOT (L2), uric acid, bilirubin total, cholesterol (L1), total protein and albumin the Sigma values were found to be between 3 and 6 .

For parameters like SGPT (L1), SGOT (L1), Creatinine (L1) and Cholesterol (L2), the sigma values were found to be less than 3. Diagram 1 and 2 displays average Sigma performance of level 1 and level 2 control respectively for one-year duration. Percent bias, TEa and Sigma values of concerned parameters for 12 months was depicted in Table 1.

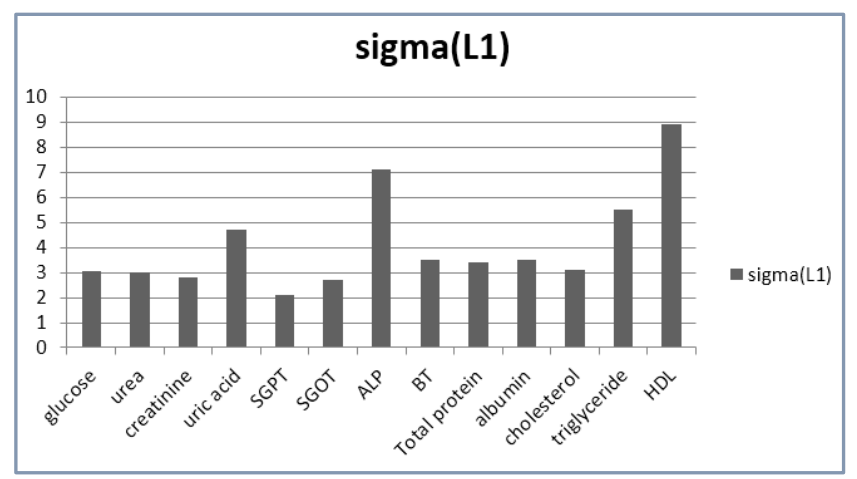

Diagram 1. Shows Average Sigma Performance of Level 1 Control for One Year

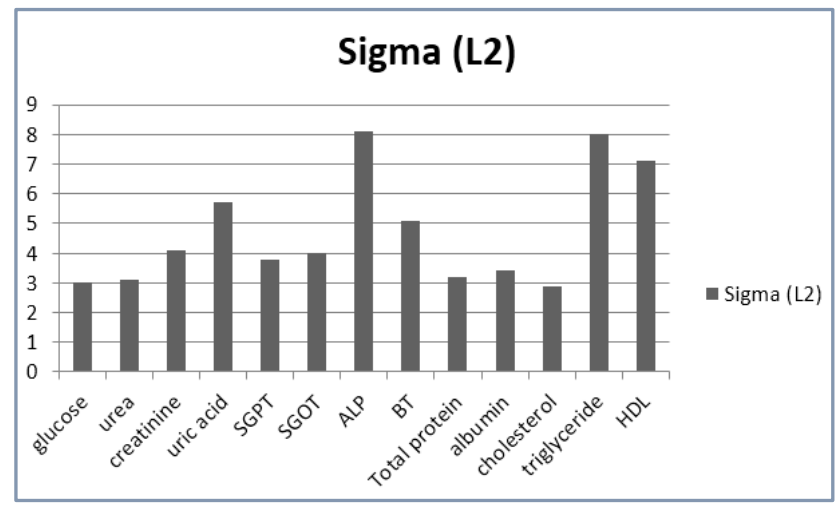

Diagram 2. Average Sigma Values of Parameters for High Control (L2)

\begin{tabular}{|l|l|l|l|l|l|l|}
\hline Parameter & $\begin{array}{l}\% \text { bias } \\
\text { (Avg) }\end{array}$ & $\begin{array}{l}\text { Total } \\
\text { allowable } \\
\text { error }\end{array}$ & $\begin{array}{l}\text { Level 1 } \\
\text { control } \\
\text { CV\% } \\
\text { (Avg) }\end{array}$ & Sigma & $\begin{array}{l}\text { Level 2 } \\
\text { control } \\
\text { CV\% } \\
\text { (Avg) }\end{array}$ & Sigma \\
\hline GLUCOSE & 0.23 & $10 \%$ & 3.2 & 3.05 & 3.20 & 3.02 \\
\hline UREA & 0.24 & $9 \%$ & 3 & 3.0 & 2.8 & 3.1 \\
\hline CREATININE & 0.58 & $15 \%$ & 4.9 & 2.9 & 3.5 & 4.1 \\
\hline URIC ACID & 0.44 & $17 \%$ & 3.5 & 4.7 & 2.9 & 5.7 \\
\hline SGPT & 0.60 & $20 \%$ & 9.2 & 1.9 & 5.1 & 3.8 \\
\hline SGOT & 0.41 & $20 \%$ & 7.2 & 2.7 & 4.8 & 4.0 \\
\hline ALP & 0.51 & $30 \%$ & 4.1 & 7.8 & 3.5 & 8.42 \\
\hline BT & 0.37 & $20 \%$ & 5.63 & 3.5 & 3.85 & 5.1 \\
\hline $\begin{array}{l}\text { TOTAL } \\
\text { PROTEIN }\end{array}$ & 0.35 & $10 \%$ & 2.8 & 3.4 & 3.07 & 3.21 \\
\hline ALBUMIN & 0.72 & $10 \%$ & 2.6 & 3.5 & 2.79 & 3.43 \\
\hline CHOLESTEROL & 0.505 & $10 \%$ & 3 & 3.1 & 3.2 & 2.16 \\
\hline TRIGLYCERIDE & 0.25 & $25 \%$ & 4.5 & 5.5 & 3.06 & 8.0 \\
\hline HDL & 0.4 & $30 \%$ & 3.3 & 8.9 & 4.2 & 7.14 \\
\hline
\end{tabular}

Table 1. \% Bias TEa and Sigma values of concerned Parameters for 12 Months

\section{DISCUSSION}

The quality of healthcare has been a primary concern of worldwide. Unlike other quality initiatives utilised by the healthcare sector like TQM six sigma is different, because it provides sustained strategic achievements with long-lasting benefits.

A sigma value indicates the frequency of defects occurring in a process. A higher sigma value translates in lower defects and a lower sigma value means a higher number of defects.

According to a study done by Usha $\mathrm{S}$ et al revealed that sigma values $<3$ for Urea, ALT, BD, BT, Ca, creatinine (L1) and urea, AST and BD (L2). Sigma lies between $3-6$ for Glucose, AST, cholesterol, uric acid, total protein (L1) and ALT, cholesterol, BT, calcium, creatinine and glucose (L2). Sigma was more than 6 for Triglyceride, ALP, HDL, albumin (L1) and TG, uric acid, ALP, HDL, albumin and total protein (L2).

According to them, Total error (TE) ideally should be calculated for each parameter using formula Total error $(\mathrm{TE})=1.96 * \mathrm{CV} \%+$ Bias \%. If Total error (TE) is less than Total allowable error (TEa), we can consider the process satisfactory.

TEa observed < TEa (CLIA) or close to it the quality requirement is met, and instrument is suitable for measurement of analyte. Only analytes for which TEa observed > TEa (CLIA) were ALT, BD, $\mathrm{Ca}+2$, creatinine (L1) 
suggesting that respective methodologies need a thorough evaluation. ${ }^{6}$

Similar findings in our study for $<3$ sigma values of ALT and creatinine level one support their conclusion regarding implementation of ideal analytical methodologies for these analytes.

Lakshman $M$ et al found that sigma metrics of Triglycerides, Lactate, Uric acid, AST, Urea, Creatine kinase (CK), Phosphate, Total Bilirubin are the best performers and the sigma value was more than 6.0 in both normal and abnormal levels, ${ }^{7}$ whereas we found triglyceride and ALP being the best performer.

The difference in our findings may be due to differences in calculating sigma using TEa from different guidelines. In their study, they used Total allowable errors (TEa) for calculating the sigma metrics from the guidelines of Dr. Carmen Ricos and her colleagues and CLIA guidelines were used by us.

According to study done by Singh Bhawna et al they obtained sigma 6 for TG, CPK-Total and Amylase for both the levels of QC. This implies that the analytical method in use is appropriate for detecting both low and high values.

In both the studies, the sigma value of one parameter which was in accordance with our result is triglyceride. They also opined that parameters which demonstrated wide variation in the sigma values for both the levels of $\mathrm{QC}$ should be evaluated with discretion. The methodology should be reevaluated.

There is also an exigency to strictly follow Westgard multi rules as well as increase the number of QC runs. Blood urea being the worst performer in their laboratory, directing special attention to this parameter is mandatory for revamping performance and it is also of utmost importance to explore urea method. 8

In our study, we found creatinine (L1), SGPT (L1) and SGOT (L1) being the worst performer. We also require upgraded analysers and better methodologies to achieve desired acceptable sigma values for these parameters.

In industries outside healthcare, 3 Sigma is considered the minimal acceptable performance for a process. When performance falls below 3 Sigma, the process is considered to be essentially unstable and unacceptable. ${ }^{3}$

For a method with Sigma below 3 calls for improvement in the method as quality of the test cannot be assured even after repeated QC runs. Thus, sigma metrics values are useful in setting the internal QC acceptability criteria. ${ }^{9}$

The term probability of false rejection (Pfr) is used to describe a situation where there are no analytical errors present except for the inherent imprecision or random error of the method. Probability of error detection (Ped) is the term used to describe where analytical error occurs in addition to the inherent random error.

For achievement of world class quality, it is desirable to have a high probability of error detection and a low probability of false rejection. ${ }^{10}$ For improvement of quality of sigma matrices, it is necessary to detect probability of false rejection. According to study in France clinical laboratories provides an incentive to develop real tools to measure performance and methods to optimise the management of internal quality controls. Their study discusses the primary factors that influence the sigma index (the choice of the total allowable error, the approach used to address bias) and compares the performance of different analysers on the basis of the sigma index. Six sigma strategy can be applied to the policy management of internal quality control in a laboratory and demonstrates through a comparison of four analysers that there is no single superior analyser in clinical chemistry. This strategy can be used in our study also, but due to lack of different types of autoanalyser it would not be possible for us to compare different instrument sigma values. The main difficulty in using the six sigma methodology lies in the absence of official guidelines for the definition of the total error acceptable. ${ }^{11}$

We have analysed 13 analytes over a period of 12 months (July 2016 - July 2017) and assessed for sigma metrics. We have only 3 analytes ALP, Triglyceride (L2) and HDL that shows satisfactory sigma value, i.e. more than 6 .

It has been demonstrated that most of the analytes are in the range of 3 - 6 sigma value, which emphasises close monitoring and improvisation of existing quality control strategy. We have sigma values $<3$ for parameters like SGPT (L1), SGOT (L1), Creatinine (L1) and Cholesterol (L2). Sigma value $<3$ may be due to reagent deterioration, poor instrument performance or methodology used.

A very stringent internal $\mathrm{QC}$ has to be undertaken for these parameters and the frequency of internal QC run should be increased as well as corrective action should be taken for these parameters. It is also important to implement appropriate QC strategies in order to augment existing Quality management system of our laboratory.

\section{CONCLUSION}

Sigma metrics will facilitate the initiation of ideal analytical methodologies in order to augment laboratory performance. Most of the laboratories demonstrated that $<3$ sigma values for parameters such as creatinine (L1) and ALT (L1) ought to be re-evaluated in search for an ideal analytical methodology, so that all the laboratories across the country could achieve identical and improved quality performance.

Most of the parameters that demonstrated sigma 3 to 6 sigma fall within acceptable sigma range with a chance for improvisation which may include improved $\mathrm{CV} \%$, Total error $<$ TEa, better method, better instrument, no. of quality control run and reduced bias \%.

Triglyceride being the best performer in sigma scale ( 6 sigma) in most of the laboratories as well as in our laboratory leads to a satisfactory quality achievement. It also brings us to the conclusion that ideal quality strategy was used for triglyceride.

Sigma matrix will help laboratories to implement correct quality control strategy which in turn will not only reduce the cost of test procedure, but also improve patient compliance.

\section{REFERENCES}

[1] Gami B, Patel D, Chauhan K, et al. Sigma metrics as a quality marker for analyzing electrolytes in laboratory. International Journal of Advanced Research 2013;1(7):197-201. Journal homepage: http://www.journalijar.

[2] Revere L, Black K. Integrating six Sigma with total quality management: a case example for measuring medication errors. J Healthcare Management 2003;48(6):377-91. 


\section{Jemds.com}

[3] Westgard S, Westgard QC. Six Sigma metric analysis for analytical testing processes. Abbott MS-09-7907. 2009;4.

[4] Kalra J, Kopargaonkar A. Quality improvement in clinical laboratories: a six Sigma concept. Path Lab Open Med Journal 2016;1(1):11-20. Article Ref. \#: 1000PLMOJ1104.

[5] U.S. Department of Health and Human Services. Clinical Laboratory Improvement Amendments of 1988. Final rules and notice. 42 CFR Part 493. Federal Register 1992;57:7188-288.

[6] Adiga US, Preethika A, Swathi K. Sigma metrics in clinical chemistry laboratory - a guide to quality control. Al Ameen J Med Science 2015;8(4):281-7.

[7] Lakshman M, Reddy BR, Bhulaxmi P, et al. Evaluation of sigma metrics in a medical biochemistry lab. International Journal of Biomedical Research 2015;6(03):164-71.

\section{Original Research Article}

[8] Singh B, Goswami B, Gupta VK, et al. Application of Sigma metrics for the assessment of quality assurance in clinical biochemistry laboratory in India: a pilot study. Ind J Clin Biochem 2011;26(2):131-5.

[9] Westgard J0. Quality control. How labs can apply six Sigma principles to quality control planning. Clin Lab News 2006;32:10-12.

[10] Westgard JO, Groth T, Aronsson T, et al. Performance characteristics of rules for internal quality control: probabilities for false rejection and error detection. Clin Chem 1977;23(10):1857-67.

[11] Scherrer F, Bouilloux JP, Calendini O, et al. Interest and limits of the six Sigma methodology in medical laboratory. Annales de Biologie Clinique (Paris) 2017;75(1):107-13. 